\title{
ON SELF-ADJOINT LINEAR RELATIONS
}

\author{
Péter Berkics ${ }^{1, *}$
}

\author{
${ }^{1}$ University of Pécs, Ifjúság útja 6, H-7624 Pécs, Hungary \\ Communicated by László Tóth \\ Original Research Paper \\ Received: Sep 16, 2020 - Accepted: Sep 22, 2020 \\ First published online: Mar 29, 2021 \\ ๑ 2020 The Author(s)
}

\begin{abstract}
A linear operator on a Hilbert space $\mathbb{H}$, in the classical approach of von Neumann, must be symmetric to guarantee selfadjointness. However, it can be shown that the symmetry could be omitted by using a criterion for the graph of the operator and the adjoint of the graph. Namely, $S$ is shown to be densely defined and closed if and only if $\left\{k+l:\{k, l\} \in G(S) \cap G(S)^{*}\right\}=\mathbb{H}$.

In a more general setup, we can consider relations instead of operators and we prove that in this situation a similar result holds. We give a necessary and sufficient condition for a linear relation to be densely defined and self-adjoint.
\end{abstract}

\section{KEYWORDS}

linear operator, graph of operator, linear relation, adjoint of a linear relation, multi-valued linear operator, von Neumann theorem

MATHEMATICS SUBJECT CLASSIFICATION (2020)

Primary 47A06; Secondary 47A05,47B25,47B65

\section{INTRODUCTION}

Self-adjoint operators on a Hilbert space is an old research topic and very important in many applications. There are well-known old results about them. Recently it turned out that classical theorems can be generalized and even reversed. The new results are quite surprising and we will state them. In this research note we give a generalization of the Stone Lemma and another equivalent properties for self-adjointness of operators.

One can see that this machinery can be treated in the more general language of linear relations. A natural question is that what properties are valid and how we can state the classical theorems in this new language.

Relations are quite basic notion and well studied in mathematics. They have a crucial role in many topics. It turns out that multi-valued operators can be represented as linear relations. The research of linear relations, as generalization of operators, was started by Richard Arens in 1961 [1], who laid the foundation of the operational calculus of linear relations. It is nowadays an active research topic. There are recent generalizations of classical theorems like the von Neumann theorem which states that for a linear operator $T$, the operator $T^{*} T$ is self-adjoint (see in [4]) and more and more basic results of functional analysis are formulated on linear relation language.

\footnotetext{
${ }^{\text {*} C o r r e s p o n d i n g ~ a u t h o r . ~ E-m a i l: ~ b e r k i c s p @ g a m m a . t t k . p t e . h u ~}$
} 
The generalization of operator theory results - like the von Neumann Theorem and the Stone lemma - on linear relation language has a fairly young history. In the late seventies T. Kato ( [3], [2]) considered unbounded self-adjoint extensions of the sum of two self-adjoint operators in a Hilbert space. To treat this problem, he introduced the concept of form sum. Also, the concept of linear relation arises in the investigation of self-adjoint extensions of the sum of two unbounded self-adjoint operators in a Hilbert space $\mathbb{H}$. The sum is not necessarily a densely defined nonnegative operator on the intersection of the domains, and even the closure of the sum is not necessarily an operator. On the other hand, the sum operator is nonnegative and it has a self-adjoint extension in $\mathbb{H}$ in the sense of multivalued operators i. e. linear relations.

Our goal in this research note is to generalise some basic statements on self-adjoint operators for linear relations. We give a more general Stone lemma and formulate an equivalent condition for self-adjointness of a linear operator on a Hilbert space. After that, we also generalize these formulated theorems for linear relations.

The structure of this note is the following. In Section 2 we remind the classical Stone lemma and von Neumann Theorem and give the reversed von Neumann Theorem and a generalization of the classical Theorem. In Section 3 we give an introduction to the notion of linear relations and its properties, and generalize the main Theorems of Section 2 for linear relations.

\section{CLASSICAL STONE LEMMA AND VON NEUMANN THEOREM AND THEIR GENERALIZ- ATIONS}

In this section we recall the classical Stone lemma and von Neumann Theorem about self-adjoint operators. Both of them have generalizations and the von Neumann Theorem even has a reverse statement. At the end of the section we give an equivalent condition for self-adjointness without the explicit statement of the symmetry.

First let us recall the definition of the graph of an operator. Let $\mathbb{H}$ a Hilbert space, $T$ a linear operator on $\mathbb{H}$.

DEFINITION 2.1. We define the graph of the operator $T$ as:

$$
G(T)=\{\{f, T f\}: f \in \operatorname{dom}(T)\},
$$

and the adjoint of the graph as:

$$
G(T)^{*}=\left\{\left\{f, f^{\prime}\right\}:\left\{f, f^{\prime}\right\} \in \mathbb{H} \times \mathbb{H},\left\langle f^{\prime}, h\right\rangle=\langle f, T h\rangle \text { for all }\{h, T h\} \in G(T)\right\} .
$$

Obviously, if $T^{*}$ exists, then $G\left(T^{*}\right)=G(T)^{*}$.

DEFINITION 2.2. An operator has full range if $\operatorname{ran}(S)=\mathbb{H}$ and it is symmetric if $G(S) \subset G\left(S^{*}\right)$.

LEMMA 2.3. (Stone Lemma, see [7] and [6]) Let $S: \mathbb{H} \rightarrow \mathbb{H}$ be a (not necessarily densely defined) symmetric linear operator. If $S$ has full range then the adjoint $S^{*}$ exists and $S=S^{*}$.

We can generalise the Stone lemma without the explicit useage of symmetry as follows:

THEOREM 2.4. Let $S: \mathbb{H} \rightarrow \mathbb{H}$ be a linear operator such that $\operatorname{ran}\left(G(S) \cap G(S)^{*}\right)=\mathbb{H}$. Then the adjoint $S^{*}$ exists and $S=S^{*}$.

Proof. Let $T$ be an appropriate operator such that $G(T)=G(S) \cap G(S)^{*}$. Then $T \subset S$ and $S^{*} \subset T^{*}$.

If $h, g \in \operatorname{dom}(T)$, then $\{g, T g\}$ and $\{h, T h\} \in\left(G(S) \cap G(S)^{*}\right)$. Therefore $T g=S g,\{g, S g\} \in G(S)^{*}$, $T h=S h$ and $\{h, S h\} \in G(S)^{*}$.

Now we show that $T$ is symmetric:

$$
\langle T g, h\rangle=\langle S g, h\rangle=\langle g, S h\rangle=\langle g, T h\rangle .
$$

Since $T$ is symmetric and has full range, we can apply the Stone lemma for the operator $T$ and we get that $T$ is self-adjoint. As a consequence, $S^{*}$ exists and $S=S^{*}$.

THEOREM 2.5. ( Classical von Neumann Theorem, see in [8]) If $T$ is densely defined and closed operator on $\mathbb{H}$, then $T^{*} T$ is a self-adjoint operator on $\mathbb{H}$.

REMARK 2.6. For a simple proof of this Theorem see [5] Chapter 3. 
THEOREM 2.7. (Revised (more general) von Neumann theorem, see in [6] Chapter 3) Let $\mathbb{H}$ and $\mathbb{K}$ be both real or complex Hilbert spaces and let $T: \mathbb{H} \rightarrow \mathbb{K}$ be a densely defined operator. The following assertions are equivalent:

(1) $T$ is a closed operator,

(2) $T^{*} T$ and $T T^{*}$ are (positive) self-adjoint operators.

The next Theorem characterizes a densely defined operator with the intersection of its graph and the adjoint of its graph.

THEOREM 2.8. Let $S$ be a positive linear operator on the Hilbert space $\mathbb{H}$. Then the following are equivalent:

(1) $\operatorname{dom}(S)^{\perp}=0$ and $S^{*}=S$

(2) $\left\{k+l:\{k, l\} \in G(S) \cap G(S)^{*}\right\}=\mathbb{H}$

Proof.

$(1) \rightarrow(2):$

$\{k+l:\{k, l\} \in G(S)\}=\{k+S k: k \in \operatorname{dom}(S)\}=\operatorname{ran}(I+S)=\mathbb{H}$.

$(2) \rightarrow(1):$

We will prove this direction in 3 steps.

(a) $g \in \operatorname{dom}(S)^{\perp}$ :

In this case we know that $\{0, g\} \in G\left(S^{*}\right)$ and there exists $\{k, l\} \in G(S) \cap G\left(S^{*}\right)$ such that $g=k+l$. Therefore $l=S k, g=k+S k$ and $\langle S h, k\rangle=\langle h, l\rangle$ for all $h \in \operatorname{dom}(S)$. As a consequence,

$$
0=\langle g, k\rangle=\langle k+S k, k\rangle=\langle k, k\rangle+\langle S k, k\rangle .
$$

Moreover $\langle S k, k\rangle \geq 0$ and $\langle k, k\rangle \geq 0$, so $k=0$ and $g=0$.

Finally we get that $\operatorname{dom}(S)^{\perp}=\{0\}$ and there exists $S^{*}$ and $G(S)^{*}=G\left(S^{*}\right)$.

(b) $g \in \operatorname{dom}\left(S^{*}\right)$ :

In this case there exists $\{k, l\} \in G(S) \cap G\left(S^{*}\right)$ such that $g+S^{*} g=k+l$, where $l=S k=S^{*} k$, therefore $g+S^{*} g=k+S^{*} k$. As a consequence we have $(g-k)+S^{*}(g-k)=0$, so $(g-k) \in$ $\operatorname{ker}\left(I+S^{*}\right)=(\operatorname{ker}(I+S))^{\perp}=(\operatorname{ran}(I+S))^{\perp}=\{0\}$.

Finally $g=k, g+S^{*} g=k+S k=g+S g$, that is $S^{*} g=S g$, so $S^{*} \subseteq S$.

(c) $g \in \operatorname{dom}(S)$ :

In this case there exists $\{k, l\} \in G(S) \cap G\left(S^{*}\right)$ such that $g+S g=k+l$, where $l=S k=S^{*} k$, therefore $g+S g=k+S k$. As a consequence, $(g-k)+S(g-k)=0$, so $(g-k) \in \operatorname{ker}(I+S)$ and

$$
\langle(g-k)+S(g-k), g-k\rangle=\langle g-k, g-k\rangle+\langle S(g-k), g-k\rangle=0 .
$$

We also know the fact that $\langle g-k, g-k\rangle \geq 0$ and $\langle S(g-k), g-k\rangle \geq 0$. Hence $g-k=0$ so $g=k$ and $g+S g=k+S^{*} k=g+S^{*} g$, that is $S g=S^{*} g$ thus $S \subseteq S^{*}$.

In summary, with the help of (a), (b) and (c) we proved that the second condition implies that $S$ is densely defined and self-adjoint.

\section{LINEAR RELATIONS}

In this section we give a short introduction to the topic of linear relations. Linear relations are natural representational forms for multivalued operators. First let us show the classical definition of linear relations on a Hilbert space.

DEFINITION 3.1. Let $\mathbb{H}$ be a Hilbert space. We call $T$ a linear relation on $\mathbb{H}$ if $T$ is a linear subspace of $\mathbb{H} \times \mathbb{H}$.

Define the domain, range, kernel and multi-value of $T$ as follows:

- $\operatorname{dom}(T)=\left\{f \in \mathbb{H}:\left\{f, f^{\prime}\right\} \in T\right\}$,

- $\operatorname{ran}(T)=\left\{f^{\prime} \in \mathbb{H}:\left\{f, f^{\prime}\right\} \in T\right\}$,

- $\operatorname{ker}(T)=\{f \in \mathbb{H}:\{f, 0\} \in T\}$,

- $\operatorname{mul}(T)=\left\{f^{\prime} \in \mathbb{H}:\left\{0, f^{\prime}\right\} \in T\right\}$. 
The previous definition gives the usual characteristics of the linear relation $T$. However, the next definition treats $T$ from a different point of view.

DEFINITION 3.2. Let $\mathbb{H}$ be a Hilbert space, $T$ a linear relation on $\mathbb{H}$ and $x \in \mathbb{H}$. Then $T(x)=\{y \in$ $\mathbb{H}:\{x, y\} \in T\}$. In this case the domain, range, and multi-value of $T$ can be defined as follows:

- $\operatorname{dom}(T)=\{x \in \mathbb{H}: T(x)$ is not empty $\}$,

- $\operatorname{ran}(T)=\bigcup_{x \in \operatorname{dom}(T)} T(x)$,

- $\operatorname{mul}(T)=T(0)$.

If $T(x)$ never contains more than one element then we call it single valued. In this case $T$ is the graph of an operator.

The linearity of $T$ can be characterized by the following expression:

$$
\alpha T\left(x_{1}\right)+\beta T\left(x_{2}\right) \subseteq T\left(\alpha x_{1}+\beta x_{2}\right) \text { for all } \alpha, \beta \text { scalar and } x_{1}, x_{2} \in \mathbb{H}
$$

DEFINITION 3.3. Let $T$ and $S$ be two linear relations on $\mathbb{H}$. The sum of $T$ and $S$ is also a linear relation on $\mathbb{H}$ :

$$
T+S=\{\{k, l+m\} \in \mathbb{H} \times \mathbb{H}:\{k, l\} \in T,\{k, m\} \in S\} .
$$

Let us define the product of two linear relations as follows:

DEFINITION 3.4. Let $T$ and $S$ be two linear relations $\mathbb{H}$. Then the product of the linear relations $S$ and $T$ is also a linear relation on $\mathbb{H}$ :

$$
\{\{k, l\} \in \mathbb{H} \times \mathbb{H}:\{k, x\} \in T,\{x, l\} \in S \text { for some } x \in \mathbb{H}\} .
$$

Now we give the definition of the adjoint of a linear relation.

DEFINITION 3.5. Let $\mathbb{H}$ be a Hilbert space and $T$ a linear relation on $\mathbb{H}$. Then the adjoint of $T$ denoted by $T^{*}$ is also a linear relation on $\mathbb{H}$ and is defined as follows:

$$
T^{*}=\left\{\left\{f, f^{\prime}\right\} \in \mathbb{H} \times \mathbb{H}:\left\langle f^{\prime}, h\right\rangle=\left\langle f, h^{\prime}\right\rangle \forall\left\{h, h^{\prime}\right\} \in T\right\} .
$$

It is important to mention that if $T$ is an operator, we can only define the adjoint if it is densely defined. However, if $T$ is a linear relation, we can always define the adjoint.

The notion of self-adjointness and positivity is nearly the same as in the operator case, however the definition of symmetry is a bit different.

DEFINITION 3.6. Let $\mathbb{H}$ be a Hilbert space and $T$ be a linear relation on $\mathbb{H}$. Then:

(1) We call $T$ self-adjoint if $T=T^{*}$.

(2) We call $T$ positive if $\left\langle f^{\prime}, f\right\rangle \geq 0$ for all $\left\{f, f^{\prime}\right\} \in T$.

(3) We call $T$ symmetric if $T \subseteq T^{*}$.

DEFINITION 3.7. Let $\mathbb{H}$ be a Hilbert space and $T$ a linear relation on $\mathbb{H}$. Let the inner product be linear in the first variable and conjugate linear in the second entry (i. e. $\langle\lambda x, y\rangle=\lambda\langle x, y\rangle$ és $\langle x, \lambda y\rangle=\bar{\lambda}\langle x, y\rangle)$

Then we can define the inner product on $T$ with the use of inner product on $\mathbb{H}$ :

$$
\langle\{k, l\},\{x, y\}\rangle=\langle k, l\rangle+\langle x, y\rangle \quad\{k, l\},\{x, y\} \in T .
$$

DEFINITION 3.8. The inverse of the linear relation $T$ can be defined as follows:

$$
T^{-1}\{\{y, x\} \in \mathbb{H} \times \mathbb{H}:\{x, y\} \in T\} .
$$

DEFINITION 3.9. We call the linear relation $T$ closed if $T$ is a closed subspace of $H \times H$.

THEOREM 3.10. Let $T$ be a positive self-adjoint linear relation. Then $\operatorname{ran}(I+T)=\mathbb{H}$.

This statement appeared in [4] without proof.

Proof. Let us show that the following holds:

$$
\{0\}=\operatorname{ker}(I+T)=\operatorname{ker}\left(T+T^{*}\right)=\operatorname{ker}\left[(I+T)^{*}\right]=[\operatorname{ran}(I+T)]^{\perp}
$$

Consider the sequences $\left(f_{n}\right)$ and $\left(f_{n}^{\prime}\right)$ such that $\left\{f_{n}, f_{n}^{\prime}\right\} \in T$ and $\left(f_{n}+f_{n}^{\prime}\right) \rightarrow g \in \mathbb{H}$. 
Then $\left\{f_{n}, f_{n}+f_{n}^{\prime}\right\} \in I+T$ and

$$
\left\langle f_{n}, f_{n}+f_{n}^{\prime}\right\rangle=\left\langle f_{n}, f_{n}\right\rangle+\left\langle f_{n}, f_{n}^{\prime}\right\rangle \geq\left\langle f_{n}, f_{n}\right\rangle
$$

Since $T$ is positive,

$$
\begin{aligned}
\left\langle f_{n}+f_{n}^{\prime}, f_{n}+f_{n}^{\prime}\right\rangle & =\left\langle f_{n}, f_{n}\right\rangle+\left\langle f_{n}, f_{n}^{\prime}\right\rangle+\left\langle f_{n}^{\prime}, f_{n}\right\rangle+\left\langle f_{n}^{\prime}, f_{n}^{\prime}\right\rangle \\
& \geq\left\langle f_{n}, f_{n}\right\rangle+\left\langle f_{n}^{\prime}, f_{n}^{\prime}\right\rangle \geq\left\langle f_{n}, f_{n}\right\rangle
\end{aligned}
$$

Let $f_{m}$ and $f_{m}^{\prime}$ be other elements of the sequence above. Then

$$
\left\langle f_{m}-f_{n}, f_{m}-f_{n}\right\rangle \leq\left\langle\left(f_{m}+f_{m}^{\prime}\right)-\left(f_{m}^{\prime}+f_{n}\right),\left(f_{m}+f_{n}^{\prime}\right)-\left(f_{n}+f_{m}^{\prime}\right)\right\rangle
$$

The right hand side of this inequality tends to zero, as $m$ and $n$ tends to infinity. Moreover, $f_{n} \rightarrow f,\left(f_{n}+f_{n}^{\prime}\right) \rightarrow g$. Therefore $\{f, g\} \in I+T$ so $f \in \operatorname{dom}(T)=\operatorname{dom}(I+T)$.

Let us summarize the known results of linear relations on a Hilbert space $\mathbb{H}$.

THEOREM 3.11. Let $\mathbb{H}$ be a Hilbert space and $T$ a linear relation on $\mathbb{H}$. Then the following hold:

(1) $(\operatorname{dom}(T))^{\perp}=\operatorname{mul}\left(T^{*}\right),(\operatorname{ran}(T))^{\perp}=\operatorname{ker}\left(T^{*}\right)$.

(2) $T^{*}$ is a closed linear relation.

(3) $T^{* *}$ is the closure of $T$ so $T \subseteq T^{* *}$. Moreover, if $T$ is closed then $T=T^{* *}$.

(4) The linear relation $T$ is single valued (in this case it is a graph of an operator) if and only if $(\operatorname{dom}(T))^{\perp}=\{0\}$.

(5) If $T$ is a symmetric linear relation on $\mathbb{H}$ and $\operatorname{dom}(T)=\mathbb{H}$ then $T$ is a graph of a bounded self-adjoint operator.

(6) If $T$ is a symmetric linear relation on $\mathbb{H}$ and $\operatorname{ran}(T)=\mathbb{H}$ then $T$ is a self-adjoint linear relation on $\mathrm{H}$.

(7) If $J: \mathbb{H} \times \mathbb{H} \rightarrow \mathbb{H} \times \mathbb{H}, J(\{f, g\}):=\{g,-f\}$, then $T^{*}=J T^{\perp}=(J T)^{\perp}$.

(8) If $T$ is a positive self-adjoint linear relation on $\mathbb{H}$ then $\operatorname{ran}(I+T)=\mathbb{H}$ where $I$ the graph of the identity operator.

(9) Let $S$ and $T$ be two linear relations on $\mathbb{H}$. Then $S \subseteq T$ implies $T^{*} \subseteq S^{*}$.

(10) $\left(T^{-1}\right)^{*}=\left(T^{*}\right)^{-1}$.

REMARK 3.12. The claims (1), (5), (6), (7), (8) have been proven in [4] and claims (2), (3), (4), (9), (10) have been proven in [1].

THEOREM 3.13. Let $T$ and $S$ be two linear relations on $\mathbb{H}$. If the kernel and range of $T$ and $S$ are equal, then $S \subseteq T$ if and only if $S=T$. [1]

THEOREM 3.14. (Generalization of von Neumann Theorem, [4].) Let $T$ be a closed linear relation on $\mathbb{H}$. Then the product $T^{*} T$ and $T T^{*}$ are a positive self-adjoint linear relations on $\mathbb{H}$.

The next theorem is the reformulation of the general Stone lemma for linear relatons.

THEOREM 3.15. Let $\mathbb{H}$ be a Hilbert space and $T$ a linear relation on $\mathbb{H}$. If $\operatorname{ran}\left(T \cap T^{*}\right)=\mathbb{H}$ then $T$ is self-adjoint.

Proof. Let us consider $S=T \cap T^{*}$.

(a) First we verify that $T \subseteq T^{*}$ :

Let $\left\{g, g^{\prime}\right\} \in T$. Then there exists $\left\{h, h^{\prime}\right\} \in S$ such that $g^{\prime}=h^{\prime}$. Therefore $\{g-h, 0\} \in T^{*}$ and there exists $\left\{k, k^{\prime}\right\} \in S$ such that $k^{\prime}=g-h$.

Moreover $\langle g-h, g-h\rangle=\left\langle k^{\prime}, g-h\right\rangle=\langle k, 0\rangle$, so $h=g$.

Finally,

$$
\left\{g, g^{\prime}\right\}=\left\{h, g^{\prime}\right\}=\left\{h, h^{\prime}\right\} \in S=T \cap T^{*} \subseteq T^{*}
$$

so $\left\{g, g^{\prime}\right\} \in T^{*}$, therefore $T \subseteq T^{*}$.

(b) Now we verify that $T^{*} \subseteq T$ :

Let $\left\{g, g^{\prime}\right\} \in T^{*}$. Then there exists $\left\{h, h^{\prime}\right\} \in S$ such that $g^{\prime}=h^{\prime}$. Now we have $\{g-h, 0\} \in$

$T^{*}$ and there exists $\left\{k, k^{\prime}\right\} \in S$ such that $k^{\prime}=g-h$.

Therefore $\langle g-h, g-h\rangle=\left\langle k^{\prime}, g-h\right\rangle=\langle k, 0\rangle$ so $h=g$. 
Finally

$$
\left\{g, g^{\prime}\right\}=\left\{h, g^{\prime}\right\}=\left\{h, h^{\prime}\right\} \in S=T \cap T^{*} \subseteq T,
$$

so $\left\{g, g^{\prime}\right\} \in T$. As a consequence $T^{*} \subseteq T$.

Summarising (a) and (b) we get that $T=T^{*}$.

THEOREM 3.16. Let $\mathbb{H}$ be a Hilbert space and $T$ a positive linear relation on $\mathbb{H}$. Then the following are equivalent:

(1) $(\operatorname{dom}(T))^{\perp}=0$ and $T^{*}=T$,

(2) There exists an operator such that $G(S)=T \cap T^{*}$ and $\operatorname{ran}(I+S)=\mathbb{H}$.

Proof.

$(1) \rightarrow(2)$

$T=G(S)$ i.e. $S$ is a positive self-adjoint operator. As a consequence $\operatorname{ran}(I+S)=\mathbb{H}$.

$(2) \rightarrow(1):$

Now $S$ is a positive symmetric operator, therefore $(\operatorname{dom}(S))^{\perp}=\{0\}$. Then $S$ is a positive selfadjoint operator.

a) $g \in\left(\operatorname{dom}(T)^{\perp}\right)$ :

There exists $h \in \operatorname{dom}(S) \subseteq \operatorname{dom}(T)$ such that $g=h+S h$.

We know that $h \in \operatorname{dom}(T)$ therefore

$$
0=\langle g, h\rangle=\langle h, h\rangle+\langle S h, h\rangle .
$$

We also know that $S$ is positive i.e. $\langle S h, h\rangle$ so $h=0$.

Finally the linearity of $S$ implies that $g=0$ and as a consequence $T$ is densely defined.

b) $\{0, g\} \in T^{*}$ :

$0=\left\langle h^{\prime}, 0\right\rangle=\langle h, g\rangle$ for all $\left\{h, h^{\prime}\right\} \in T$ therefore $g \in(\operatorname{dom}(T))^{\perp}$. As a consequence $T^{*}$ is the graph of an operator.

c) $g \in \operatorname{dom}\left(T^{*}\right)$ :

There exists $h \in \operatorname{dom}(S)$ such that $g+T^{*} g=h+S h$. We know that $G(S) \subseteq T^{*}$ therefore $(g-h)+T^{*}(g-h)=0$.

Then $(g-h) \in \operatorname{ker}\left(I+T^{*}\right)=\operatorname{ker}\left((I+T)^{*}\right)=(\operatorname{ran}(I+T))^{\perp} \subseteq(\operatorname{ran}(I+S))^{\perp}=\{0\}$.

As a consequence $T^{*} g=S h=S g=T g$ therefore $g=h \subseteq \operatorname{som}(S) \subseteq \operatorname{dom}(T)$.

With this, we proved that $T^{*} \subseteq T$.

d) $g \in \operatorname{dom}(T)$ :

There exists $h \in \operatorname{dom}(S)$ such that $g+T g=h+T h$.

We know that $G(S) \subseteq T$ so $(g-h)+T(g-h)=0$.

We also know that $T$ is positive so $\langle T(g-h), g-h\rangle \geq 0$.

$$
\begin{aligned}
\langle 0, g-h\rangle & =\langle(g-h)+T(g-h), g-h\rangle \\
& =\langle g-h, g-h\rangle+\langle T(g-h), g-h\rangle
\end{aligned}
$$

i.e. $g=h \subseteq \operatorname{dom}(S) \subseteq \operatorname{dom}\left(T^{*}\right)$.

So we proved that $T \subseteq T^{*}$.

Summarizing the previous points we get that $T$ is a densely defined self-adjoint operator.

REMARK 3.17. The previous result not only gives us another equivalent condition for a linear relation to be self-adjoint, but also shows that if the second condition holds, then $S$ will be the graph of an operator.

\section{ACKNOWLEDGEMENTS}

The author is grateful to Zoltán Sebestyén for his great research seminars and suggestions. He also thanks the referee for useful comments. 


\section{REFERENCES}

[1] Arens, R. Operational calculus of linear relations. Pacific f. Math. 11, 1 (1961), 9-23.

[2] Kato, T. Trotter's product formula for an arbitrary pair of self-adjoint contraction semigroups. In Topics in Functional Analysis, vol. 3 of Advances in Mathematics Supplementary Studies. 1978, pp. $185-195$.

[3] Kato, T. Perturbation Theory for Linear Operators. Springer-Verlag, Berlin, 1980.

[4] SAndovici, A. Von neumann's theorem for linear relations. Linear and Multilinear Algebra (2017). doi:10.1080/03081087.2017.1369930.

[5] Sebestyén, Z., AND TARCSAy, Z. Characterization of self-adjoint operators. Studia Scientiarum Mathematicarum Hungarica 50, 4 (2013), 423-435. doi:10.1556/SScMath.50.2013.4.1252.

[6] Sebestyén, Z., And Tarcsay, Z. A reversed von neumann theorem. Acta Scientiarum Mathematicarum (Szeged) 80, 3-4 (2014), 659-664.

[7] Stone, M. Linear transformations in Hilbert space, vol. 15 of American Mathematical Society Colloquium Publications. American Mathematical Society, Providence, Rhode Island, 1932.

[8] von Neumann, J. Über adjungierte funktionaloperatoren. The Annals of Mathematics 33, 2 (1932). 294. Crossref. Web.

Open Access statement. This is an open-access article distributed under the terms of the Creative Commons Attribution 4.0 International License (https://creativecommons.org/licenses/by/4.0/), which permits unrestricted use, distribution, and reproduction in any medium, provided the original author and source are credited, a link to the CC License is provided, and changes - if any - are indicated. (SID_1) 